\title{
Modeling Competition-Driven Business Strategy for Business IT Alignment
}

\author{
Constantinos Giannoulis ${ }^{1}$, Michaël Petit ${ }^{2}$, and Jelena Zdravkovic ${ }^{1}$ \\ 1 Department of Computer and Systems Sciences, Stockholm University \\ Forum 100, SE-164-40 Kista, Sweden \\ constantinos, jelenaz@dsv.su.se \\ 2 PReCISE Research Center, Computer Science Department, University of Namur \\ Rue Grandgagnage 21, B-5000 Namur, Belgium \\ mpe@info.fundp.ac.be
}

\begin{abstract}
Business strategy aims at supporting the vision of an enterprise, by paving the way to achieve it through goals that direct the strategy's execution. Aligning business strategy to system requirements requires explicit models from both business strategy and requirements engineering. However, existing business strategy definition approaches are informal and their syntax is based on natural language, therefore, they cannot be used in model-driven alignment. An objective of our research is to define a well-structured business strategy modeling language. In this paper, we propose a business strategy meta-model based on Porter's work on competition driven strategy and its extension by Stabell and Fjeldstad. Our UML meta-model is formalized in Telos and OWL. An initial validation is performed by instantiating the meta-model using a case scenario.
\end{abstract}

Keywords: business strategy, alignment, meta-modeling, Telos, OWL.

\section{Introduction}

Enterprises constantly aim at enabling the communication of business strategy, typically by linking decision makers and executives with practitioners and employees, and product and service offers with concrete tasks [1. Such links are necessary to align people, products and services with long-term visions, and further, to help in ensuring that IT systems are defined and designed in accordance to business strategy, thereby contributing to solve the always-present problem of business-IT alignment [2].

Within the Information Systems modeling community, business intentions and following strategies are typically conceptualized using goal-based modeling approaches, such as in Goal-Oriented Requirements Engineering (GORE) [3]. The capabilities of the those approaches are challenged when capturing and expressing abstract business notions and interrelations such as the ones defined by business strategy. On the other side, the generality of these notions, constitutes

C. Salinesi and O. Pastor (Eds.): CAiSE 2011 Workshops, LNBIP 83, pp. 16 28, 2011.

(C) Springer-Verlag Berlin Heidelberg 2011 
a challenge for their representational capabilities at a conceptual level; existing business strategy frameworks provide rich expressiveness but are not formal and precise. In [4] we have elicited the gap between business strategy and requirements for system development. Additionally, we argued that a formal conceptualization of business strategy becoming the interface to GORE contributes towards the improvement of business-IT alignment.

In our research, we aim at unifying rigorously business strategy concepts proposed by approaches rooted in Strategic Management (e.g. (Mintzberg's ten schools of thought on strategy [5]), into an ontology. Gradually, we aim to examine noteworthy business strategy definition approaches and create their representations in an ontology to provide a unified business strategy meta-model (UBSMM) with applicability for practice. Our mode of work was inspired by the Unified Enterprise Modeling Language (UEML) approach aiming at a framework for interoperability of enterprise modeling languages by defining a core language for enterprise and IS modeling [6]. In previous work [47, we have conceptualized Strategy Maps [1] and Balanced Scorecards (BSC) [9] by the means of a meta-model and we have also formalized the ontological base using OWL [10] and Telos [11.

In this study, we extend our modeling efforts towards competition driven strategy approaches, as proposed by the work of Porter named Value Chain [12 13 14], further extended by Stabell and Fjeldstad with the Value Shop and the Value Network configurations 15. To the best of our knowledge no similar effort exists, including both modeling and formalizing the Value Chain, Shop and Network. Following a similar methodological path as in [7], in this study, we have first conceptualized a meta-model to capture business strategy defined using the aforementioned value configurations. Then we have formalized the metamodel to the ontology level, to improve both its quality (such as unambiguity and model-consistency checking) and usability in the model-driven development context (automatic process-ability). We chose to formalize the strategy metamodel in two complementary ontology languages, Telos [11] and OWL [10, having different expressiveness, tool support and capabilities. To demonstrate the applicability and validity of the modeled value-based business strategy, we have considered a real case scenario, which captures an experience report from the application of the Value Shop at the Norwegian Police [16].

The paper is structured as follows: Section 2 presents Porter's positioning strategy approach extended beyond the Value Chain to include the Value Shop and the Value Network as well as the Norwegian Police case scenario [16] used later to validate our contribution. Section 3 motivates modeling business strategy from an RE perspective and presents our contribution: an extended conceptualization of the value configuration (Porter, Stabell and Fjeldstad), as well as its ontological definition with OWL and Telos. Section 4 illustrates the validation of the meta-model using the Norwegian Police case scenario by prototype implementation using Telos and OWL support tools. Section 5 provides our conclusions and directions for further work. 


\section{Business Strategy}

Strategic planning is the process during which a strategy is defined by analyzing the current status of the enterprise and the competitive environment in which it resides. Within the premises of this generic definition of strategic planning, prevalent approaches defining business strategy include; Miles et al. [17, Desarbo et al [18, Blue Ocean Strategies [19], Strategy Maps 20] \& Balanced Scorecards [9] and the Value Chain [12] extended by Stabell and Fjeldstad [15]. In [47], as part of our incremental effort, we have conceptualized Strategy Maps \& Balanced Scorecards which utilize the three views identified by Barney's grouping of strategic planning approaches [21]: resource-based view, Schumpeterian view, industrial organization. In the remaining of this section we present the Value Chain, and its extensions of Stabell and Fjeldstad, which are conceptualized in section 3 .

\subsection{Porter vs Stabell and Fjeldstad}

According to Porter: "Strategy is the creation of a unique and valuable position involving a different set of activities." 1214. Strategic positioning requires establishing and maintaining six essential principles: the right goal, a value proposition, a distinctive value chain, trade-offs, fit and continuity [14]. The value chain [12] (figure 1(a) reflects how an organization structures its activities to offer the unique value differently from any competitor and highlights a company's strategy and strategy implementation depending on how activities for production are carried out. It consists of value activities and margin. Value activities capture everything an organization does to create value for its buyers, divided into primary and support, while margin is the difference between the total value and the total cost of performing the value activities. Primary activities are the ones involved in a product's creation, sale, and transfer and post-sale assistance. Support activities provide procured inputs, technology, human resources, and general company support across the organization.

Each activity is classified based on its contribution to a firm's competitive advantage, primarily from an economic view; those that have high impact of differentiation and those that have a considerable proportion of cost. Porter identifies ten generic drivers for cost and value [12, which shape the competitive position of the firm: scale, capacity utilization, linkages, interrelationships, vertical integration, location, timing, learning, policy decisions and government regulations. Value activities interact with each other within the value chain via linkages, which are relationships between the way a value activity is performed and the cost of another (e.g. the dotted lines in figure 1(a) . They support optimization and coordination among value activities, thus competitive advantage. Linkages may exist between multiple value chains (e.g. firm and suppliers). All support activities except from firm infrastructure can directly be linked to specific primary activities as well as support the whole value chain, whereas firm infrastructure cannot but only support the value chain in its totality. According to Porter, success in a competitive environment accompanied with an enterprise's desired 


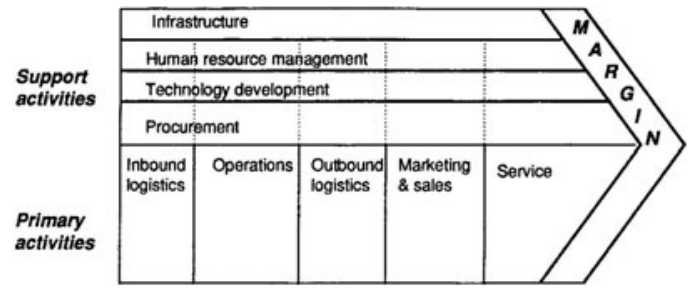

(a) The Value Chain [12].

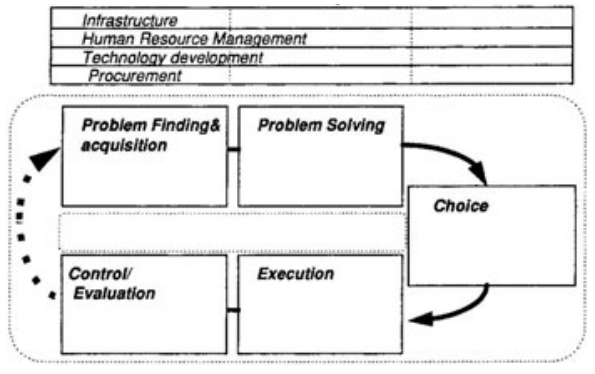

(b) The Value Shop [15].

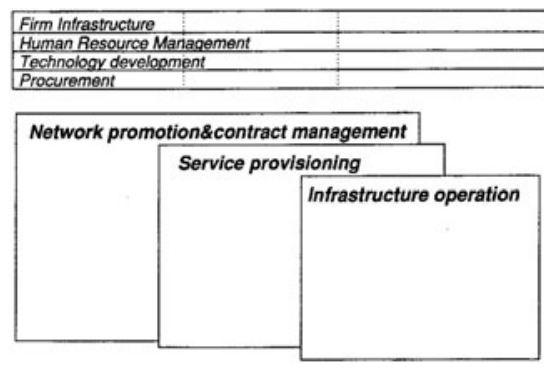

(c) The Value Network [15.

Fig. 1. The Value Configurations (a) (b) and (c)

targeted market segment results into three generic strategies; cost leadership, differentiation and focus 12 .

Stabell and Fjeldstad [15] introduced the choice of value configuration to move beyond production and extend the value chain to the value shop, where value is created by using resources and activities to resolve a customer problem as well as to the value network, where value is created by facilitating relationships among a network of enterprises and their customers via a mediating technology. In a value shop the primary value activities include; problem finding and acquisition, problem solving, choice, execution, control and evaluation (figure $1(\mathrm{~b})$ ). In a value network the primary value activities include; network promotion and contract management, service provisioning and infrastructure operation (figure 1(c)).

A fundamental difference among the value configurations lies on the value creation logic; the value chain focuses on transforming inputs into outputs, the value shop focuses on resolving customer problems and the value network focuses on linking customers. Additionally, value activities within a value chain are linked sequentially (suppliers, producers, and distributors) by adding value to what the preceding activity has produced, within a value shop value activities are linked spirally interchanging problem-solving and implementation activities, and finally, within a value network value activities are linked simultaneously and in parallel forming horizontally interconnected activities. For the remaining of the paper, we will be referring to the value chain, the value shop and the value network as value configuration. 


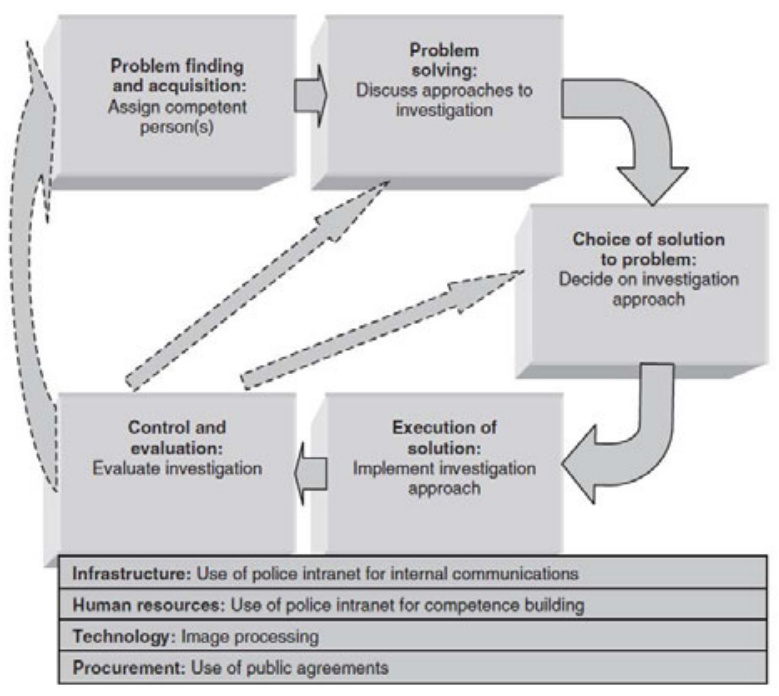

Fig. 2. Police investigation unit as value shop with activity examples [16]

\subsection{Case Scenario: A Value Shop for police investigation}

Gottschalk and Berg [16] have applied the value configuration of the value shop to evaluate police investigation performance for investigations that use information systems. The case scenario does not capture the strategy of the whole organization, it rather captures the strategic intent of a unit, investigation, where value is created by solving unique problems. Figure 2 illustrates the example of a police investigation unit as a value shop with concrete activities introduced. Solid arrows represent the sequential execution of activities while dotted arrows represent the cyclical execution.

\section{Modeling Competition-Driven Business Strategy}

This section presents our contribution, a conceptualization of value configurations into a well defined meta-model. The first part motivates how modeling business strategy contributes to business-IT alignment, the second part presents our meta-model, the third part presents the formalization using Telos and OWL.

\subsection{Modeling of Business Strategy for Alignment}

The increased availability of IT means for facilitating the businesses of enterprises, requires their alignment with business strategy to make them operational and also justify investments on IT 1 . A common approach is to map business

\footnotetext{
${ }^{1}$ This paper addresses business strategy from the scope of Strategic Management
} independent of business-IT alignment approaches. 


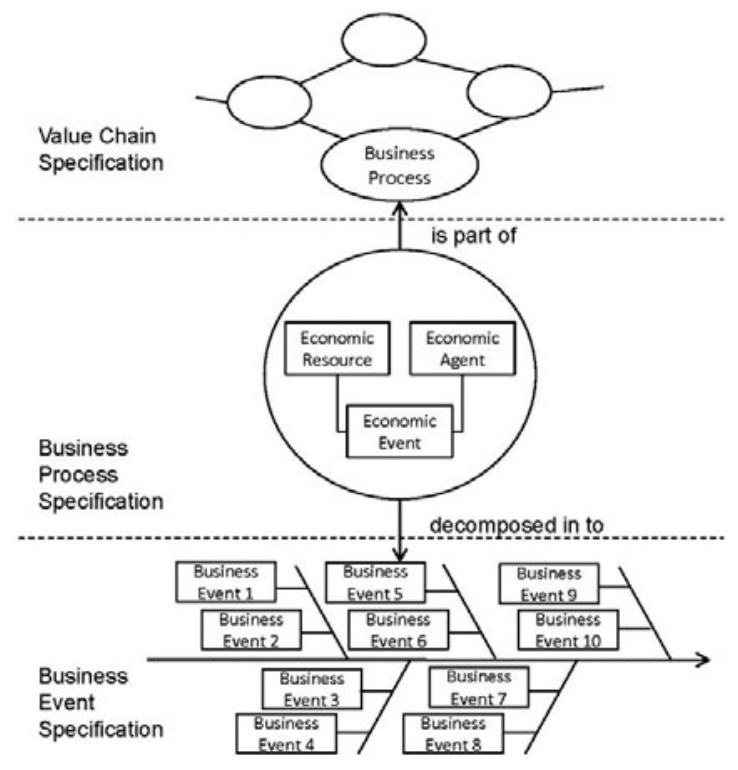

Fig. 3. Value driven alignment [27]

strategy to enterprise models and then further to system requirements. Goaloriented enterprise models are often used in this context 2223]. Obtained goal models are then, using GORE, transformed to requirements for systems.

A fundamental notion for value configurations is that a product or a service gains a value as it passes through a stream of business activities of an enterprise. Thereby, it seems reasonable to refine a strategy defined using a value configuration approach, by exploring it using value-centric enterprise models such as business value models. Notable examples of business-value frameworks are REA [24, BMO 25] and $\mathrm{e}^{3}$ value [26].

In [27, the REA framework has been extended to capture value analysis starting from Porter's value chain (figure 3). At the top, an enterprise value chain is defined as a series of connected business processes, where each process is adding value towards customers and corresponds to value activities. For example, in the "Find competent persons" value process shown in figure 3, the inputs will be the people and means responsible for finding the persons competent for investigations, and the output, the commitment of the persons found towards the investigations of interest. Each process in an enterprise value chain is decomposed to the next level (Business Process Specification) by exploring at least two economic events related to the process: a decrement event that consumes the input resource and an increment event that acquires the output resource. "Find competent persons" process includes a "recruit" decrement event and a "contract obtainment" increment event. Once all economic events issuing 
the exchange of economic resources are elicited, they may be decomposed into workflows of low-level tasks, i.e. business events. These workflows correspond to business processes, which involve the tasks that operationalize consumption and acquisition of resources, such as announcing the positions for investigators, contacting candidates, analyzing obtained applications, and so forth. A value configuration strategy decomposed in the described way typically results in a large number of operational business processes. The models obtained can be further transformed to high-level system requirements, which may facilitate goals of individual process tasks or a group of tasks using IT means.

In 28, the described REA framework is considered as the starting model in an MDA-like approach for designing e-services and further Web services. In [29], the authors elaborate an approach, based on requirements engineering techniques, to design e-services from $\mathrm{e}^{3}$ value business models. The approach is model-driven and considers three vertical/successive perspectives in design: value perspective (from the business model), process perspective (primary, management and support business processes are captured) and information systems perspective (Web services, BPEL orchestrations). Since any of these approaches rely on a value analysis using business models, adding the strategy analysis dimension on top of the business modeling is of an important benefit; additionally, having this dimension modeled in a formal and precise way as we propose in this paper, contributes to some of the major principles of model-driven development, as advocated in 30 .

\subsection{The Value Configuration Meta-model}

A meta-model defines the conceptual elements of a language, their possible interrelations and constraints on possible syntactically correct combinations of these elements and relations 31 (also echoed by 32). We have defined our meta-model through inference from the value chain, value shop and value network found in the studied literature 12 13 14 15. Our meta-model, formalized in UML, is presented in figure 4. Constraints define the allowed interrelations and imposed restrictions for each construct. Some constraints are represented as cardinalities on relationships and others captured in natural language are presented later in this section. Given the ambiguity of value configurations and textual descriptions, we have considered and analyzed them all, to obtain a precise and complete formalization. Due to space limitations some generalizations are not shown in figure 4. A complete version of the value configuration meta-model including all specializations can be found on the authors' web-page 2 .

Classes, relationships and cardinalities: Strategy captures the desired strategic positioning of an organization and according to 14 should include a long term goal, the value proposition, which appear as attributes.

Value Configuration captures the value configuration of an organization that supports the strategy and can be either a value chain or a value shop or a value network. It includes a description and a margin.

2 http://www.info.fundp.ac.be/ mpe/BUSITAL2011 


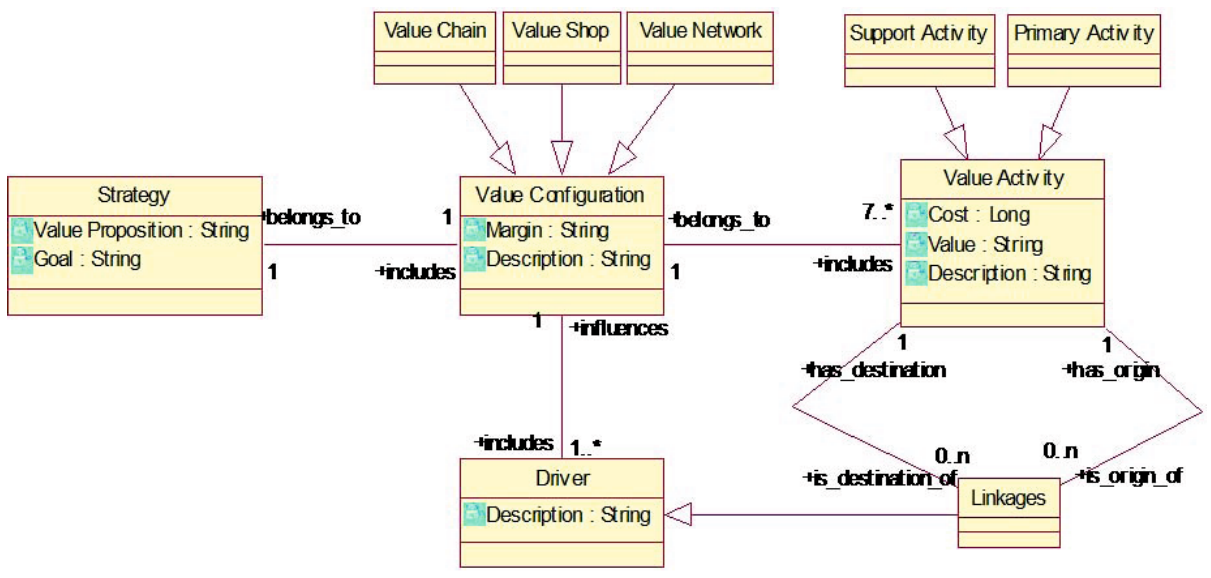

Fig. 4. The Value Configuration Meta-model

Driver captures all parameters influencing cost and value in a value configuration and includes a description as attribute but also 10 specializations (scale, capacity, utilization, linkages, interrelationships, vertical integration, location timing, learning, policy decisions and government regulations). Due to space limitations only linkages are shown in figure 4 as they are of significant importance, which represent the links between Value Activities.

Value Activity captures the activities that constitute a value configuration of an organization and is specialized into Primary Activity and Support Activity while it includes cost, value and description as attributes. Support Activity is further specialized into four common classes to all value configurations: infrastructure management, HR management, procurement, technology development. Primary Activity is also further specialized into: (i) Value Chain Primary Activity, further specialized to all value chain primary activities: Inbound Logistics, Operations, Service, Marketing \& Sales, Outbound Logistics. (ii) Value Shop Primary Activity, specialized to all value shop primary activities: Problem Solving, Choice, Execution, Problem finding \& Acquisition, Control \& Evaluation and Value Network Primary Activity. (iii) Value Network Primary Activity, specialized to all value network primary activities: Infrastructure Operation, Service Provisioning, Network Promotion \& Contract Management.

Constraints: Specialization hierarchies are modeled as partitions; an instance of a parent class must be an instance of exactly one child class. Value configurations include at least one instance of each type of support activity. Primary activities must be of the appropriate type (e.g. primary activities in a Value Network are all belonging to the class of value network primary activities). A value configuration of a particular type includes at least one primary activity of each relevant type (e.g. a value network includes at least one activity of network promotion, one activity of service provisioning and one of infrastructure operation. Each SupportActivity is origin of at least one Linkage. Each PrimaryActivity is 
destination of at least one Linkage whose origin is a SupportActivity. A Linkage links two different value activities.

\subsection{Formalization}

The meta-model presented in the previous section is meant to be input for a unified business strategy meta-model (UBSMM), integrated with our Strategy Map and Balanced Score Card meta-model [7. Therefore, the meta-model should be as precise and validated as possible. In this direction, we have formalized our meta-model because (i) the process of formalizing the meta-model itself helps to improve its quality because it raises questions that would not otherwise be asked; (ii) formal semantics of the languages used for formalization allow to discover properties of the meta-model (consistency, un-anticipated properties, etc.); (iii) the non-ambiguity of formalisms results in a clear and unambiguous abstract syntax for our UBSMM; (iv) formal languages are often equipped with tools that support the expression and analysis of the meta-model, as well as other operations on meta-models helpful in future steps of our research (particularly in integration); (v) using standard languages for formalization can help both in diffusion and re-usability of our UBSMM.

Considering the aforementioned benefits, we chose to formalize our metamodel in two complementary languages with different expressiveness, tool support and capabilities, Telos and OWL. The complete Telos and OWL models, as well as the relevant code, can be downloaded from the authors' web-page 3 . OTelos, a dialect of Telos, is a classical object-oriented deductive knowledge representation language, particularly suited for meta-modeling, supported by the Conceptbase [33. We have used the language's expressive and simple syntax to formalize the advanced constraints that apply to our meta-model. We have used Conceptbase to check the consistency of the set of constraints by instantiating the meta-model with particular elements from our case scenario. However, Conceptbase and Telos do not provide elaborate built-in capabilities to reason about the meta-model as well as integrate several meta-models. The formalization in OWL, using Protégé [34, serves as a basis for future more thorough analysis and integration of meta-models. We have transformed our UML class diagram into an OWL implementation based on the rules found in 35. Constraints, are captured through OWL restrictions within the documented limitations of the language (e.g. derivation is not directly supported by OWL [35]). OWL allows for more advanced reasoning by checking that the ontology makes sense (satisfiability), finding implicit terminology (e.g. subsumption) and finding implicit facts, etc. 36. Additionally, OWL has the advantage of being more widely accepted and used, and supported by numerous freely available tools.

\section{Validation}

In this section we illustrate the applicability and validity of our meta-model using Telos and OWL for the application of the Value Shop at a police investigation

\footnotetext{
3 http://www.info.fundp.ac.be/ mpe/BUSITAL2011
} 


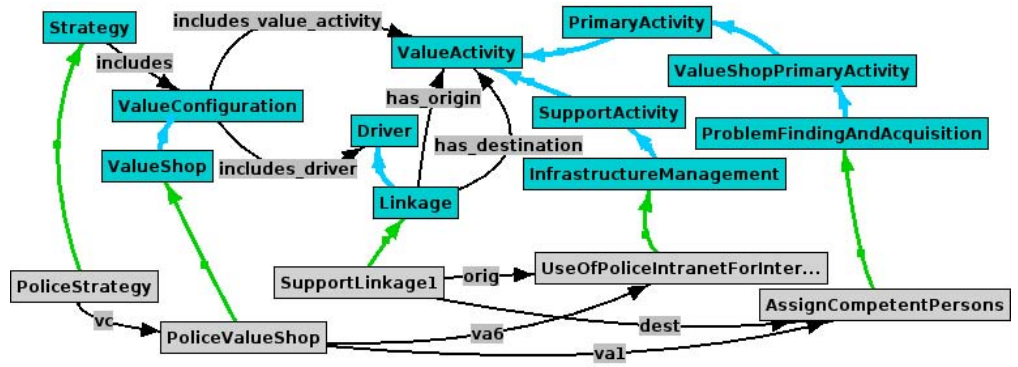

Fig. 5. The Value Shop in Telos: the Police Investigation Unit

unit (figure 2] section 2.2) and due to space limitations we present an extract from Telos (complete Telos and OWL instantiation models, can be downloaded from the authors' web-page 3 ).

The model was produced using mapping rules from UML, similar to the ones used in [7. Constraints described in section 3.2 were formalized and attached to the relevant classes. Figure 5 shows an extract of the Telos meta-model for the case. Boxes on top represent classes of the meta-model and boxes on the bottom instances from the case, which are related through Telos attributes classes corresponding to the UML associations, specialization links and instantiation relationships (arrows). The figure shows that the Police ValueShop includes amongst others two value activities, namely AssignCompetentPersons, an instance of ProblemFindingAndAcquisition, itself a subclass of PrimaryActivity. This activity is supported through a linkage by the UseOfPoliceIntranetForInternalCommunications activity which is an instance of InfrastructureManagement, a subclass of SupportActivity. We formalized all constraints and instantiated the constructs one by one (e.g. starting from the concept of Strategy). When the instantiation did not result into any violation of any constraint, then we checked if it was intuitively correct (according to our understanding of the literature). If correct, we proceeded with the creation of more instances of the case. If incorrect, we refined the model, possibly by adding a missing constraint. When the instantiation resulted into the violation of a constraint, we checked if it was intuitively correct (according to our understanding of the literature). If correct, we completed the model with additional instances required to avoid the constraint violation and then tried again. We continued until all constructs of the case were instantiated. Additionally, we tested some cases that would intuitively violate the constraints and we checked whether they were not accepted. If they were, we corrected the constraints accordingly.

Finally, it should be noted that this process does not guarantee the metamodel is error free, as more test cases would be required to improve the coverage of the tests. Nevertheless, it indicates a real case can be instantiated illustrating the model is consistent and accepts at least one model, which is applicable to reality. 


\section{Conclusions and Future Work}

In this paper, we presented a step towards our objective of creation of a welldefined meta-model for modeling business strategy. We reported on the formalization of the specific set of concepts proposed by a well-know approach for strategy definition, namely the Value Chain concept proposed by Porter and its extensions by Stabell and Fjelstad. The meta-model is defined in terms of a UML class diagram but is also formalized in Telos and OWL that allow a rigorous expression of constraints. Furthermore, we used supporting tools associated with these languages to perform an initial validation of the meta-model and its constraints by showing that it can be instantiated, respecting the constraints, for a case from the literature, thus demonstrating applicability in practice.

Despite the fact we have systematically elicited the concepts from the input texts, some more fuzzy concepts have not yet been integrated. We intend to further analyze these concepts in the future and evaluate the benefits of integrating them in the meta-model. Further validation of the meta-model is obviously needed by testing it on more cases. The next steps of our research, consist of integrating our meta-model to more business strategy modeling approaches, such as [7] and start mapping them to GORE.

\section{References}

1. Kaplan, R.S., Norton, D.P.: Mastering the Management System. J. Harvard Business Review 86, 63-77 (2008)

2. Chan, Y.E., Horner, R.B.: IT alignment: what have we learned? J. Journal of Information Technology 22(4), 297 (2007)

3. van Lamsweerde, A.: Requirements Engineering: From System Goals to UML Models to Software Specifications. Wiley, West Sussex (2009)

4. Giannoulis, C., Petit, M., Zdravkovic, J.: Towards a Unified Business Strategy Language: A Meta-model of Strategy Maps. In: van Bommel, P., Hoppenbrouwers, S., Overbeek, S., Proper, E., Barjis, J. (eds.) PoEM 2010. Lecture Notes in Business Information Processing, vol. 68, pp. 205-216. Springer, Heidelberg (2010)

5. Mintzberg, H., Ahlstrand, B., Lampel, J.: Strategy Safari: The complete guide through the wilds of strategic management. Prentice Hall, London (1998)

6. Anaya, V., Berio, G., Harzallah, M., Heymans, P., Matulevicius, R., Opdahl, A., Panetto, H., Verdecho, M.J.: The Unified Enterprise Modeling Language Overview and further work. J. Computers in Industry 61 (2009)

7. Giannoulis, C., Petit, M., Zdravkovic, J.: Modeling Business Strategy: A Metamodel of Strategy Maps and Balance Scorecards. In: 5th IEEE International Conference on Research Challenges in Information Science (RCIS 2011). IEEE, New York (2011) (accepted)

8. Kaplan, R.S., Norton, D.P.: Having trouble with your strategy? Then map it. J. Harvard Business Review 78(5), 167-176 (2000)

9. Kaplan, R.S., Norton, D.P.: The Balanced Scorecard: translating Strategy into Action. Harvard Business School Press, Boston (1996)

10. Smith, M., Welty, C., McGuinness, D.: OWL Web Ontology Language Guide, http://www.w3.org/TR/2003/WD-owl-guide-20030331 
11. Mylopoulos, J., Borgida, A., Jarke, M., Koubarakis, M.: Telos: A Language for Representing Knowledge About Information Systems. J. ACM Transaction on Information Systems 8(4), 325-362 (1990)

12. Porter, M.E.: Competitive Advantage: Creating and Sustaining Superior Performance. Free Press, New York (1985)

13. Porter, M.E.: Competitive Strategy: Techniques for Analyzing Industries and Competitors. Free Press, New York (1998)

14. Porter, M.E.: On Competition. Harvard Business School Publishing, Boston (2008)

15. Stabell, C.B., Fjeldstad, Ø.D.: Configuring Value for Competitive Advantage: On Chais, Shops, and Networks. J. Strategic Management 19(5), 413-437 (1998)

16. Gottschalk, P., Holgersson, S.: Stages of Knowledge management technology in the value shop: the case of police investigation performance. J. Expert Systems 23(4), 183-193 (2006)

17. Miles, R.E., Snow, C.C., Meyer, A.D., Coleman, H.J.: Organizational strategy, structure, and process. J. Academy of Management Review 3(3), 546-562 (1978)

18. DeSarbo, W.S., Benedetto, C.A., Sinha, I.: Revisiting the Miles and Snow strategic framework: uncovering interrelationships between strategic types, capabilities, environmental uncertainty, and firm performance. J. Strategic Management 26(1), 47-74 (2005)

19. Mauborgne, R., Kim, W.C.: Blue Ocean Strategy: From Theory to Practice. J. California Management Review 47(3), 105-122 (2005)

20. Kaplan, R.S., Norton, D.P.: The strategy map: guide to aligning intangible assets. J. Strategy \& Leadership 32(5), 10-17 (2004)

21. Barney, J.: Types of Competition and the Theory of Strategy: Toward an Integrative Framework. J. Academy of Management Review 32(11), 1231-1241 (1986)

22. Bleistein, S.J., Cox, K., Verner, J.: Validating strategic alignment of organizational IT requirements using goal modeling and problem diagrams. J. Systems and Software 79, 362-378 (2006)

23. Babar, A., Zowghi, D., Chew, E.: Using Goals to Model Strategy Map for Business IT Alignment. In: 5th International Workshop on Business/IT Alignment and Interoperability (BUSITAL 2010), pp. 16-30 (2010)

24. McCarthy, W.E.: REA Accounting Model: A Generalized Framework for Accounting Systems in a Shared Data Environment. J. The Accounting Review 57(3), 554-578 (1982)

25. Osterwalder, A. (2004). The Business Model Ontology. Doctoral thesis, HEC Lausanne (2004), http://www.hec.unil.ch/aosterwa/ (retrieved May 02, 2009)

26. Gordijn, J.: E-Business Modeling Using the e3 Value Ontology. In: Curry, W. (ed.) E-Business Model Ontologies, pp. 98-128. Elsevier, Butterworth-Heinemann, UK (2004)

27. Geerts, G., McCarthy, W.E.: An Ontological Analysis of the Primitives of the Extended-REA Enterprise Information Architecture. The International Journal of Accounting Information Systems 3, 1-16 (2002)

28. Gordijn, J., van Eck, P., Wieringa, R.: Requirements Engineering Techniques for e-Services. In: Georgakopoulos, D., Papazoglou, M.P. (eds.) Service-Oriented Computing: Cooperative Information Systems Series, pp. 331-352. The MIT Press, Cambridge (2008)

29. Zdravkovic, J., Ilayperuma, T.: A Model-driven Approach for Designing E-Services Using Business Ontological Frameworks. In: 14th IEEE International EDOC Conference, pp. 121-130. IEEE Computer Society, Los Alamitos (2010)

30. Selic, B.: The pragmatics of model-driven development. IEEE Software 20, 19-25 (2003) 
31. Harel, D., Rumpe, B.: Meaningful Modeling: What's the Semantics of "semantics"? J. Computer 37, 64-72 (2004)

32. Lucena, M., Santos, E., Silva, C., Alencar, F., Silva, M.J., Castro, J.: Towards a unified metamodel for i*. In: 2nd International Conference on Research Challenges in Information Science (RCIS 2008), pp. 237-246. IEEE Press, New York (2008)

33. Conceptbase: A Database System for Meta-modeling and Method Engineering (tool), http://conceptbase.sourceforge.net/ (last accessed 2010-11-20)

34. Protégé Ontology Editor. Open source tool, http://protege.stanford.edu/ (last accessed 2010-11-20)

35. Ontology Definition Meta-model (ODM): Object Management Group (2009)

36. Calvanese, D., Giacomo, G.D., Horridge, M., Möller, R., Turhan, A.Y.: Reasoning for Ontology Engineering and Usage. In: Tutorial at ISWC 2008 (2008), http://owl.cs.manchester.ac.uk/2008/iswc-tones/ 